\title{
Chimpanzees (Pan troglodytes) display limited behavioural flexibility when faced with a changing foraging task requiring tool use
}

\author{
Rachel A Harrison ${ }^{1,2}$, Andrew Whiten ${ }^{\text {Corresp. } 1}$ \\ ${ }^{1}$ Centre for Social Learning and Cognitive Evolution, School of Psychology \& Neuroscience, University of St Andrews, St Andrews, United Kingdom \\ 2 School of Psychology, University of Birmingham, Birmingham, United Kingdom \\ Corresponding Author: Andrew Whiten \\ Email address: aw2@st-andrews.ac.uk
}

Behavioural flexibility, the ability to alter behaviour in response to environmental feedback, and to relinquish previously successful solutions to problems, is a crucial ability in allowing organisms to adapt to novel environments and environmental change; it is essential to cumulative cultural change. To explore this ability in chimpanzees, 18 individuals (Pan troglodytes) were presented with an artificial foraging task consisting of a tube partially filled with juice that could be reached by hand or retrieved using tool materials to hand. Effective solutions were then restricted in the second phase of the study by narrowing the diameter of the tube, necessitating the abandonment of previously successful solutions. Chimpanzees showed limited behavioural flexibility in comparison to some previous studies, increasing their use of effective techniques, but also continuing to attempt solutions that had been rendered ineffective. This adds to a literature reporting divergent evidence for flexibility (the ability to alter behaviour in response to environmental feedback, and to relinquish previously successful solutions to problems) versus conservatism (a reluctance or inability to explore or adopt novel solutions to problems when a solution is already known) in apes. 
3 Chimpanzees (Pan troglodytes) display limited behavioural flexibility when faced with a changing foraging task requiring tool use

\section{Rachel A. Harrison ${ }^{\mathrm{a}, \mathrm{b}}$ \& Andrew Whiten ${ }^{\mathrm{a}}$}

8

${ }^{\text {a }}$ Centre for Social Learning and Cognitive Evolution, School of Psychology \& Neuroscience, University of St Andrews, St Andrews, United Kingdom Andrews, St Andrews, United Kingdom. E-mail: aw2@st-andrews.ac.uk

Acknowledgements

We are grateful to the Royal Zoological Society of Scotland for permission to conduct this study at Edinburgh Zoo, and to the Budongo Trail keepers for all their assistance. We thank Tom comments on an earlier version of the manuscript. 


\begin{abstract}
20 Abstract
21 Behavioural flexibility, the ability to alter behaviour in response to environmental feedback, and

22 to relinquish previously successful solutions to problems, is a crucial ability in allowing

23 organisms to adapt to novel environments and environmental change; it is essential to

24 cumulative cultural change. To explore this ability in chimpanzees, 18 individuals (Pan

25 troglodytes) were presented with an artificial foraging task consisting of a tube partially filled

26 with juice that could be reached by hand or retrieved using tool materials to hand. Effective

27 solutions were then restricted in the second phase of the study by narrowing the diameter of the

28 tube, necessitating the abandonment of previously successful solutions. Chimpanzees showed

29 limited behavioural flexibility in comparison to some previous studies, increasing their use of

30 effective techniques, but also continuing to attempt solutions that had been rendered ineffective.

31 This adds to a literature reporting divergent evidence for flexibility (the ability to alter behaviour

32 in response to environmental feedback, and to relinquish previously successful solutions to

33 problems) versus conservatism (a reluctance or inability to explore or adopt novel solutions to

34 problems when a solution is already known) in apes.
\end{abstract}




\section{Introduction}

Behavioural flexibility, the ability to alter behaviour based upon environmental feedback and to inhibit previously successful behaviours, is an ability that allows organisms to adapt their behaviour to suit changing or novel environments and supports problem solving by allowing individuals to adapt their behaviour to success or failure at a problem (Sol, Timmermans \& Lefebvre 2002; Griffin \& Guez 2014; Chow, Lea \& Leaver 2016; Audet \& Lefebvre 2017). Behavioural flexibility can also describe the capacity for, and interest in, continuing to acquire novel solutions to an unchanging problem for which a solution is already known (Lehner, Burkart \& van Schaik 2011), though most experimental explorations of behavioural flexibility incorporate changes in task parameters (and therefore changes in environmental feedback). Whilst innovation (defined by Reader and Laland, 2003, pp.14, as "A process that results in new or modified learned behaviour and that introduces novel behavioural variants into a population's repertoire") has been suggested to be a component of behavioural flexibility (Lehner et al. 2011), we also consider the acquisition of novel behaviours via social learning to constitute flexible behaviour (Wright et al. 2010), and indeed the application of known behaviours to a novel problem. Behavioural flexibility is thought to be a key ability supporting the evolution of cumulative culture (Dean et al. 2014). With culture defined as "group-typical behaviour patterns shared by members of a community that rely on socially learned and transmitted information" (Laland \& Hoppitt 2003, pp.151), cumulative culture is the process whereby these socially learnt behaviours are modified and the modifications are retained, resulting in behaviours and technologies more complex than an individual could invent within their lifetime (Tomasello 1994; Tennie, Call \& Tomasello 2009). As cumulative culture relies upon the modification of known behaviours it necessitates flexibility in both the process of innovation by some individuals, and in the acquisition by others of the improved behaviours that result.

$\underline{\text { Behavioural flexibility and cumulative culture }}$

Evidence for cumulative culture in our closest living relative, the chimpanzee, is limited and controversial (Dean et al. 2014; Whiten 2017), with Boesch (2003) highlighting three behaviours observed in wild chimpanzees (nut-cracking incorporating additional stones to stabilise the anvil, parasite manipulation in which parasites are placed on a leaf which is then folded and cut, and well-digging incorporating the use of leaf-sponges to retrieve water from deep wells) as potential 
67 evidence of cumulative culture, whilst Sanz, Call and Morgan (2009) describe apparent 68 improvements made to termite fishing tools by chimpanzees in the Goualougo Triangle,

69 Republic of Congo. However, such interpretations require assumptions to be made regarding the social transmission of these behaviours, whether they represent greater complexity than that achievable by an individual alone, and indeed whether they are the result of cumulative progressions rather than unconnected innovations (which might be better described as 'accumulation'; Dean et al. 2014). With such limited evidence of cumulative culture in chimpanzees, investigation of the abilities required to support cumulative culture is required. We suggest that behavioural flexibility, along with innovation and social learning, should be considered and investigated as a potential limiting factor for cumulative culture. These three capacities are expected to work in concert to support cumulative culture - an individual innovates an improvement to a behaviour or tradition, and this improvement is passed on via social learning to other group members. As cumulative culture requires the modification of known behaviours, flexibility is required in both the innovator, to modify a behaviour within their repertoire, and in the group members socially acquiring this modified behaviour (that, in a process of cumulation, is expected to replace a known behaviour which previously served the same purpose). As outlined by Charbonneau (2015), an ability to innovate entirely novel behaviours 'from scratch' alone is not sufficient to support cumulative culture, which instead requires an ability to modify known behaviours. High-fidelity social transmission is believed to be critical in supporting cumulative culture, as it prevents backwards 'slippage' or the loss of modifications to behaviours (Tennie et al. 2009; Lewis \& Laland 2012). However, somewhat paradoxically, at least some individuals in a population must also be capable of modifying behaviours, having acquired them via high-fidelity social learning.

Measuring behavioural flexibility

Behavioural flexibility has been assessed at a species level via the proxy measure of innovation frequency (for example, Lefebvre et al. 1997; Lefebvre, Reader \& Sol 2004, for avian comparisons), and innovation has been considered a component of behavioural flexibility (Lehner et al. 2011; Audet \& Lefebvre 2017). This makes the assumption that behavioural flexibility leads to increased innovativeness, which may not always be the case at either an individual or species level (Griffin et al. 2013; Logan 2016). Behavioural flexibility in response 
97 to environmental change has been tested directly in a range of species, both in the wild and 98 captivity, frequently by employing experimental paradigms in which animals must respond to a change in task parameters, such as reversal learning (Bond, Kamil \& Balda 2007; Boogert, Monceau \& Lefebvre 2010; Manrique \& Call, 2015; Liu et al.2016) or multi-access puzzle box tests (Auersperg et al. 2011; Lehner et al.2011; Manrique, Völter \& Call 2013; Richter, Hochner \& Kuba 2016).

Both intra- and interspecific differences in flexibility have been found using these experimental techniques. Individual differences in behavioural flexibility within species have been shown in animals as diverse as octopuses, pigs and mice (Benus et al.1990; Richter et al. 2016; Bolhuis et al.2004), as well as in wild chimpanzees and sanctuary-housed orangutans (Gruber 2016). Such inter-individual differences within species are perhaps not surprising, given potential differences between individuals in terms of task motivation, cognitive ability, and personality (the latter having been shown to impact performance on problem solving tasks in chimpanzees; Massen et al., 2013). In the context of cumulative culture, an understanding of which individuals are most capable of behavioural flexibility, and investigation of any other traits that might co-occur with behavioural flexibility on an individual level, may allow predictions to be made regarding which individuals or demographics are likely to contribute to the modification of behaviours in a population's repertoire.

In addition, interspecific differences in behavioural flexibility have been demonstrated in both corvids and great apes (Bond et al. 2007; Manrique et al. 2013), and investigation of these interspecific differences allows exploration of hypotheses regarding the evolution of behavioural flexibility. Bond et al. (2007) argue that behavioural flexibility may be more apparent in species with highly complex social systems, in order to cope with rapidly fluctuating social contexts, and found that pinyon jays, a highly social species, had lower error rates on a serial reversal learning tasks than Clark's nutcrackers (a relatively solitary species with specialised spatial memory supporting caching behaviour) and western (California) scrub jays (a generalist species in terms of both ecology and social behaviour). Continued investigation of species' abilities to behave flexibly will allow investigation of the evolutionary pressures that lead to high behavioural flexibility, and will also allow investigation of other cognitive abilities that may co-evolve with behavioural flexibility. For example, it has also been shown that pinyon jays outperform Clark's nutcrackers on social learning tasks (Templeton, Kamil \& Balda 1999). Given that a suite of 
128 abilities is likely required to support cumulative culture (Dean et al. 2014), an understanding of 129 why component abilities may emerge, and whether they share common evolutionary origins, is 130 likely to enhance our understanding of the evolution of cumulative culture.

131 Observation of the tool preferences of wild tool-using species also provides some 132 indication of the flexibility of such species. Examination of the tool preferences of wild New 133 Caledonian crows found strong, persistent local preferences in terms of the plant species from 134 which their hook-tools are manufactured (St Clair et al. 2016), despite access to alternative plants, 135 which are preferred, at another, nearby study site. This may indicate some level of individual 136 conservatism (a reluctance to explore alternative solutions) in this species' tool manufacture, 137 though the authors also point out that it shows that crows at one study site have either switched 138 from a previous tool material, or acquired hooked stick tool-use relatively recently. Both 139 scenarios would reflect flexibility, both on the part of initial innovators and any individuals that 140 may have socially acquired this alternative behaviour. Study of individual crows' tool 141 manufacture indicates multiple bending techniques and adjustments during manufacture, which 142 would seem to indicate flexibility (Klump et al. 2015; Rutz et al. 2016). Wild chimpanzees have 143 been observed to modify their tool-use behaviours, with an individual in Bossou being observed 144 firstly applying a tool and technique generally used for ant-dipping on the ground to ant-fishing 145 in trees, and later using tools of a length better suited to ant-fishing in trees (Yamamoto et al. 146 2008). Wild chimpanzees have also been observed to socially learn novel tool behaviours, such 147 as crafting sponges from moss rather than leaves (Hobaiter et al. 2014). These observations of 148 wild behaviour provide some insight into chimpanzees' capacity for behavioural flexibility.

Chimpanzees - behaviourally flexible or conservative?

In several studies, chimpanzees have been reported to be conservative, rather than flexible, in their approach to artificial foraging tasks, continuing to use a habitual solution despite the prospect of gaining a greater reward via behavioural change (Marshall-Pescini \& Whiten 2008) or the habitual solution being made impossible or unrewarding (Hrubesch et al. 2009; Bonnie et al. 2012). Behavioural conservatism has also been reported in wild chimpanzees, with Gruber et al. (2009) finding that two different communities relied upon their respective habitual tool behaviours when faced with a novel artificial honey-dipping task, in one community failing to acquire the useful tool behaviour used by the other community even when scaffolded towards it 
158 (Gruber et al. 2011; Gruber 2016). Similarly, Cardoso and Ottoni (2016) found that providing

159 two communities of wild bearded capuchin monkeys with a dipping task resulted in only the

160 group that already habitually used probing tools solving the task, again despite efforts to scaffold

161 the non-probe-using group towards this behaviour. Chimpanzees may also continue to perform

162 behaviours when these behaviours are no longer necessary, for example, continuing to avoid a

163 non-functional trap in the inverted trap-tube problem (Povinelli 2000; though see also Mulcahy

$164 \&$ Call 2006), although it should be noted that in paradigms in which continuing this behaviour is

165 equally rewarding as ceasing to avoid the trap, adult humans also continue to avoid non-

166 functional traps (Silva, Page \& Silva 2005). Related to these findings of apparent conservatism

167 or lack of flexibility in chimpanzees is the concept of 'functional fixedness': the inability to

168 invent a novel use for a tool with which the animal already has experience (Hanus et al. 2011;

169 Brosnan \& Hopper 2014). Hanus et al. (2011) found that captive chimpanzees were more likely

170 to solve the 'floating peanut' experiment (in which water must be added to a tube in order to

171 raise a floating peanut to a level at which it can be reached) when a novel water dispenser was

172 added to their enclosure. The authors suggest that the old dispenser had a fixed function for the

173 animals, which prevented them from discovering it as a potential task solution.

174 By contrast, other recent studies have implied that under certain conditions, great apes

175 may be capable of flexibly altering their behaviour in response to a changing task (Lehner et al.

176 2011; Manrique et al. 2013; Yamamoto, Humle \& Tanaka 2013; Davis et al. 2016; Vale et al.

177 2017). In the studies of both Lehner et al. (2011), and Manrique et al. (2013), in order to

178 successfully retrieve a reward, great apes had to not only flexibly alter their behaviour in

179 response to changing tasks, but also develop novel methods of solving the foraging tasks that

180 they were presented with. Lehner et al. (2011) found that orangutans were capable of inventing

181 novel solutions to a task in which juice could be retrieved from a tube by dipping tool materials

182 into the tube. When the width of the tube was narrowed, orangutans flexibly altered their

183 behaviour, abandoning previously successful solutions and inventing novel solutions, some of

184 which the authors argue are cumulative improvements upon previous solutions. It should be

185 noted that although novel solutions were not experimentally seeded in the Lehner et al. study

186 (2011), orangutans were tested under group conditions, and so potentially had access to social

187 information. In a study testing all four nonhuman species of great ape, in which subjects were

188 tested individually (eliminating the possibility of social learning), Manrique et al. (2013) found 
189 that all species were able to abandon previous solutions and invent novel solutions to a non-tool-

190 based artificial foraging task in which solutions were rendered obsolete in three stages, though

191 orangutans performed more poorly than other species, only solving two stages. These studies

192 demonstrate that great apes can be capable of flexibly altering their behaviour and relinquishing

193 previously successful behaviours in order to solve changing artificial foraging tasks, without the

194 benefit of experimentally-seeded solutions.

195 Studies have also shown that chimpanzees are able to socially acquire more efficient or

196 more rewarding solutions to problems, having already mastered a less efficient solution.

197 Yamamoto et al. (2013) found that chimpanzees provided with a task in which juice could be

198 retrieved from within a tube via a small hole were capable of swapping from an inefficient

199 'dipping' technique to a more efficient 'straw-sucking' technique following observation of a

200 conspecific employing the more efficient technique, while Vale et al. (2017) found that

201 following the removal of simple tools that could be used to acquire juice in a dipping task,

202 chimpanzees began to use complex tools that required modification (unscrewing a valve so a

203 tube could be used as a 'straw' to suck up juice). Davis et al. (2016) found that chimpanzees

204 were able to relinquish a highly inefficient task solution in favour of a more efficient solution

205 when provided with demonstrations of the efficient solution by a conspecific or human

206 demonstrator. The authors of the latter study argue that such flexibility may be more apparent in

207 situations in which there is a large difference in efficiency between two technique options (with a

208 relatively highly inefficient solution more likely to be relinquished). This may suggest

209 chimpanzees use a 'copy-if-dissatisfied' strategy when given the opportunity to socially acquire

210 more efficient solutions to problems (Laland 2004). If so, behavioural flexibility in such studies

211 may be apparent only when the original solution is rendered highly inefficient and unsatisfying.

212 The findings of Lehner et al. (2011) and Manrique et al. (2013) may, in light of this, imply that

213 great apes also employ an 'explore-if-dissatisfied' strategy when given the opportunity to

214 achieve improved solutions to problems without the benefit of an existing competent model.

\section{Study aims}

216 We aimed to investigate the capability of chimpanzees to alter their behaviour in response to an

217 artificial foraging task in which viable solutions became restricted as time progressed, using a

218 liquid-retrieval task comparable to that used by Lehner et al. (2011) with orangutans. 
220 Chimpanzees were presented with the task as a group, and therefore had access to both

221

222

individual and social information about the task and potential solutions. This means that individuals were not limited to what they themselves could invent in terms of task solutions, which we believe provides a more ecologically valid measure of behavioural flexibility, as this has been defined in the past as the continued acquisition of new solutions through either innovation or social learning (Lehner et al. 2011). If chimpanzees are able to respond flexibly to changes in task conditions (as shown by Manrique et al. 2013), we would predict individuals to increase their use of techniques that remain effective, and to decrease their use of techniques that have been rendered ineffective, in the face of task restrictions. Alternatively, if chimpanzees are behaviourally inflexible, we predict the continued use of ineffective techniques and no increase in the use of effective techniques. If chimpanzees follow an 'explore if dissatisfied' strategy, we would predict the emergence of novel techniques following the imposition of task restrictions, whilst a 'copy if dissatisfied' strategy would result in the acquisition of effective techniques by individuals other than the technique's innovator following the imposition of task restrictions.

$\underline{\text { Subgroups and the impact of developmental conditions }}$

Chimpanzees were studied in Edinburgh Zoo, which houses a single community that originates from two separate subgroups; long-term residents of the zoo ('Edinburgh' chimpanzees), and others introduced in 2010 ('Beekse Bergen' chimpanzees). These subgroups are now well integrated socially (Schel et al. 2012; Watson et al. 2015) but have differing life histories, with the Beekse Bergen subgroup coming from a laboratory and first living in a zoo environment from 2007. The majority of chimpanzees in the Beekse Bergen subgroup were hand-reared in nurseries rather than mother-reared, whilst all captive-born chimpanzees in the Edinburgh subgroup were mother-reared. Both rearing history and housing have been found to impact problem solving ability (and other behaviour) in chimpanzees (Brent, Bloomsmith \& Fisher 1995; Bloomsmith et al 2006; Morimura \& Mori 2010; Vlamings, Hare \& Call 2010), so this study also considers the impact of subgroup membership upon task performance. Members of the Beekse Bergen subgroup, with a history of laboratory-housing and including a number of hand-reared chimpanzees, were predicted to perform more poorly on the task than long-term zoo residents. 
248 Scaffolding towards an effective solution

249 Learning through exposure to the artefacts of others' tool use is hypothesised to aid in the

250 development of tool behaviour in wild populations (Tennie et al. 2009); however, previous

251 experimental studies of chimpanzees (Gruber et al. 2011) and capuchin monkeys (Cardoso \&

252 Ottoni 2016) have not shown that providing scaffolding intended to approximate these artefacts

253 (tool materials already inserted into a task, for example) leads to the acquisition of novel tool

254 behaviours. By providing chimpanzees with limited exposure to scaffolding towards a novel tool

255 technique in the final phase of our study, we examined whether chimpanzees could acquire novel

256 tool behaviour through exposure to favourable affordances in the form of an effective tool

257 material correctly inserted into the task, as might occur in the wild where proficient tool-users

258 leave tools in place that novices may discover.

\section{Methods}

260 Ethical approval

261 The study received ethical approval from the University of St Andrews Animal Welfare and

262 Ethics Committee, and was approved by the Budongo Trail Research Committee. Research was

263 conducted in accordance with the guidelines of the Association for the Study of Animal

264 Behaviour.

265 Subjects and study site

266 Subjects were 18 chimpanzees housed as one group in the Budongo Trail facility at Edinburgh

267 Zoo (see Table 1 for details). The group is composed of two 'subgroups', long-term residents of

268 the zoo ('Edinburgh' chimpanzees), and others introduced in 2010 ('Beekse Bergen'

269 chimpanzees), each numbering nine individuals. The chimpanzees lived in three interconnecting

270 indoor enclosures measuring $120 \mathrm{~m}^{2}$ each and one outdoor enclosure measuring $1832 \mathrm{~m}^{2}$, along

271 with two research 'pods' (two connected rooms measuring $26.5 \mathrm{~m}^{2}$ in total) provided for

272 cognitive research. Chimpanzees were fed six to eight scatter feeds throughout the day at random

273 times and locations, with water available ad libitum throughout the facility. The research pods

274 were accessible via overhead tunnels from the chimpanzees' indoor enclosures, and activity

275 within the pods could not be viewed by chimpanzees outside the pods. Our task was presented in 
276 one of these research pods, and chimpanzees had access to both pods during testing.

277 Chimpanzees in the second research pod during testing had limited visual access to the task

278 through an open slide door separating the pods, while individuals in the primary pod (in which

279 the task was presented) generally had good visual access to the task. A maximum of seven

280 chimpanzees were recorded as present in the primary pod during testing, with space for further

281 individuals in the second pod. All chimpanzees had previous experience with artificial foraging

282 tasks involving tool use, having been provided with the 'Panpipes' task (in which a stick tool is

283 used to retrieve a grape from within the apparatus, see Whiten, Horner and de Waal, 2005, for

284 details of the apparatus) and a food-raking task in which plastic stick tools could be used to

285 retrieve out-of-reach rewards (see Price et al., 2009, for a similar task) (V West, unpublished

286 master's thesis; R Harrison, unpublished master's thesis). In addition, chimpanzees had previous

287 experience using a touchscreen device in the research pods (Wallace et al. 2017).

288 [nsert Table 1 here]

289

$\underline{\text { Apparatus }}$

290 Echoing the study of Lehner et al. (2011), we provided the chimpanzees with an artificial

291 foraging task in which dilute Ribena juice could be retrieved from within transparent

292 polycarbonate tubes, using either provided tool materials or hands. The task had two stages, for

293 which two widths of tube were provided. The first ('wide') tube measured $30 \mathrm{~cm}$ tall with a $10 \mathrm{~cm}$

294 inner diameter (see Figure 1), and the second ('narrow') tube measured $30 \mathrm{~cm}$ tall with a $5 \mathrm{~cm}$

295 inner diameter. These tubes were presented in the research pods, bolted to a polycarbonate screen

296 facing into the pod, and could be filled and emptied by the experimenter through holes in the

297 screen. Alongside the tubes, chimpanzees were provided with a selection of tool materials

298 comprising plain sticks (rigid sticks measuring approximately 40-50cm), straw bedding material,

299 strips of cloth and 'wood wool'/'excelsior' (fine wood slivers typically provided as bedding

300 material). Leafy sticks (a selection of browse generally provided to the chimpanzees by care staff

301 for nesting and feeding, primarily eucalyptus - these leafy sticks were flexible and measured

302 approximately $40-80 \mathrm{~cm}$ ) were available in the chimpanzees' enclosure throughout testing, and

303 were placed in the research pod along with the other tool materials from the fifth hour of testing

304 onwards. Chimpanzees were familiar with all tool materials, as straw, cloth, wood wool and

305 browse were generally provided as nesting material, and sticks were readily available in their 
306

307

308

309

310

311

312

313

314

315

316

317

318

319

320

321

322

323

324

325

326

327

328

329

330

331

332

333

334

335

outdoor enclosure. All tool materials were placed into the research pods before chimpanzees were given access.

\section{Procedure}

The apparatus was presented during twice-daily research sessions facilitated by Edinburgh Zoo staff. These sessions lasted for 45-60 minutes. In the first stage of the study ('Wide Tube' phase), chimpanzees were provided with the wide tube, filled with diluted sugar free Ribena to a depth of around $7 \mathrm{~cm}$. This was presented alongside the aforementioned selection of tool materials, and in the 'Wide Tube' phase the juice could also be reached by hand. All provided tool materials could potentially be used to successfully retrieve juice from the task. The tube was baited with juice for ten sessions, each lasting between 45 and 60 minutes, with juice added by the experimenter to ensure a reward was present for the duration of each session. The tube was emptied of liquid by the researcher through a valve at the base at the end of each session, as removing the chimpanzees from the research pods was logistically unfeasible, and so the juice reward was removed in order to discourage further interaction with the tube outside of the twicedaily research session.

In the second stage of the study ('Narrow Tube' phase), chimpanzees were presented with the narrow tube, filled with juice to a depth of $7 \mathrm{~cm}$, along with the same selection of tool materials. Again, chimpanzees had access to the baited tube for ten 45-60 minute sessions. The narrow tube prevented chimpanzees from inserting their hands into the tube to gain juice, and also rendered attempts made using rigid, leafless sticks relatively unsuccessful, due to the ledge surrounding the panel to which the tube was attached. This ledge meant that rigid sticks inserted into the tube frequently hit the back of the tube and could be inserted no further, preventing them from reaching the juice. Leafy sticks remained functional. Absorbent materials (straw, cloth, and wood wool) could no longer be inserted and retrieved by hand.

In the third stage of testing ('Narrow Scaffolded' phase), chimpanzees were again presented with the narrow tube for ten 45-60 minute sessions, but at the start of each day of testing, prior to the chimpanzees having access to the research pods, a leafy stick was inserted into the tube, with its leaves in the juice. As multiple sessions were conducted on some testing days, with no access to the research pods to re-insert the stick following the start of the first session each day, this provided four sessions which began with a stick already inserted. Only the 
336 first individual to interact with the task on these four sessions encountered this scaffolding;

337 however, their interactions with the scaffolding and their subsequent interactions with the task 338 could be observed by other group members.

In the course of testing, on 12 occasions, two sessions occurred on the same day, and due

340 to the impossibility of removing chimpanzees from the research pods between sessions,

341 chimpanzees had access to the empty tube and remaining tool materials for approximately one

342 hour between sessions. It was not possible to document any attempts made during this time as

343 the researcher did not have access to the adjacent research office to film or observe behaviours in

344 between sessions.

345 [insert Figure 1 here]

346 Data collection and coding

347 Sessions were recorded using a Sony Handycam DCR-SX21 camera. This recorded chimpanzees'

348 responses to the task, while the experimenter narrated chimpanzees' actions as well as the

349 identity and presence of other individuals in the research pod. The video and narration were later

350 coded together. The chimpanzee identity, tool material choice (hand, plain stick, wood wool,

351 straw, or leafy stick), action (dip, retrieve, squash, drop), and success of individuals attempting

352 the task were coded for each attempt. The presence of other individuals in the research pod was

353 also coded for each attempt. Techniques were then classified as 'Always effective' and 'Partially

354 effective' (Table 2), based upon their potential efficacy in narrow tube conditions. A second

355 coder, blind to the experimental hypotheses, coded 50 randomly selected attempts for tool

356 material, technique (as listed in Table 2), and success. Narration of these attempts did not include

357 narration of tool material, technique or success, and so the second coding was independent. Inter-

358 observer reliability was calculated using Cohen's Kappa, revealing agreement for all variables

359 (tool material $K=0.90$, technique $K=0.87$, success $K=0.95$ ).

360 Data analysis

361 Data were analysed in R (version 3.2.2, R Core Team 2015) and RStudio (version

362 0.99.893, RStudio Team, 2015) using a generalised linear mixed model with a Laplace

363 approximation (GLMM), using the function glmer in the R statistics package lme4 (Bates,

364 Maechler \& Bolker 2012). Analysing binomial data using GLMM is recommended by Jaeger 
365 (2008) and Bolker et al. (2009). The impact of predictor variables upon the number of 'Always

366 effective' vs 'Partially effective' technique attempts in the 'Wide Tube' and 'Narrow Tube'

367 phases was assessed for all individuals that made attempts in both the 'Wide' and 'Narrow' tube

368 phases $(\mathrm{N}=10)$.

369 A full random slope model was fitted (for discussion on the benefits of fitting random

370 slopes as well as random intercepts, see Schielzeth \& Forstmeier 2009). The full model

371 contained fixed effects for Phase ('Wide Tube' vs 'Narrow Tube') and Subgroup (Edinburgh vs

372 Beekse Bergen), along with an interaction between the two and a random intercept and slope by

373 Phase for each Individual $(\mathrm{N}=10)$. This full model was compared with a null model including

374 only the random intercept and slope by Phase for each Individual. Both models were fitted using

375 a binomial error structure, due to the binary nature of the response variable (Effectiveness), and a

376 logit link function. A likelihood ratio test comparing the full and null models indicated that the

377 full model was a significantly better fit $\left(\chi^{2}=8.65, d f=3, p=.0343\right.$; dAIC $\left.=2.7\right)$.

378 In addition, a full random slope GLMM was fitted to examine the likelihood of 'Always

379 effective' vs 'Partially effective' across experimental sessions within the 'Narrow Tube' phase,

380 in order to investigate any change in behaviour over time within this phase. The model contained

381 a fixed effect for Session (the ten 45-60 minute sessions that comprised each phase), and a

382 random intercept and slope by Session for each Individual that took part in the 'Narrow Tube'

383 phase $(\mathrm{N}=11)$. As with the main analysis described above, this model was compared with a null

384 model containing only the random effects, and a likelihood ratio test indicated that the model

385 containing a fixed effect for Session was no improvement upon a null model $\left(\chi^{2}=2.37, d f=1, p\right.$

$386=.1238 ; \mathrm{dAIC}=0.4)$.

\section{Results}

388

389

390

391

392

393

394

Sixteen of the eighteen chimpanzees interacted with the task during at least one of the three phases of testing (see Table 1 for frequency of participation for all individuals) In addition, sixteen of eighteen individuals were present in the research pod during another individual's attempt at the task on at least one occasion (with only Cindy and Liberius never having the opportunity to observe others at the task). Ten individuals attempted the task in both the 'Wide Tube' and 'Narrow Tube' phases. Seven individuals participated in all three phases (see Table 1). A total of 3,022 attempts were made across the three phases and 30 hours of testing. Seven 
395

396

397

398

399

400

401

402

403

404

405

406

407

408

409

410

411

412

413

414

415

416

417

418

419

420

421

422

423

424

techniques were used to successfully solve the task (see Table 2). Six of these techniques emerged in the 'Wide Tube' phase (and four of these six within the first testing session), with the remaining technique emerging in the 'Narrow Tube' phase. No novel techniques were observed in the 'Narrow Scaffolded' phase. Techniques involving the use of hands only and the use of plain, rigid sticks emerged first, within the first testing session. The use of leafy sticks emerged after several hours of exposure to the task (Table 2). Only one novel technique (wood wool retrieve) emerged in the 'Narrow Tube' phase, though some individuals performed techniques in this phase that were novel to them (though not novel to the group, and so potentially acquired socially) (Table 3).

\section{Behavioural flexibility}

In order to examine behavioural flexibility in the chimpanzees' response to the task restrictions imposed by the transition from the 'Wide Tube' to 'Narrow Tube' phase, the techniques described above were categorised as 'Always effective' or 'Partially effective' according to their potential efficacy across phases (see Table 2). Note that all techniques (see Table 2) were effective in the 'Wide Tube' phase, as the tube width allowed individuals to insert their hands into the tube, facilitating the use of a wide variety of techniques.

Techniques involving the insertion of a hand into the tube (i.e. hand dip) were classified as 'Partially effective', as insertion of the hand into the tube was made impossible by the width of the tube in the 'Narrow Tube' phase, though this technique could be successfully used in the 'Wide Tube' phases. Techniques reliant upon the insertion of a plain, rigid stick into the tube were also classified as 'Partially effective', as the overhang of the task presentation window was such that these sticks could frequently no longer be inserted into the tube in the 'Narrow Tube' phase. Short rigid sticks could still successfully be dropped into the tube and retrieved by hand, and so the technique stick drop is categorised as 'Always effective'. For further discussion of this classification, see Supplemental Article S1.

Comparison of the use of 'Partially effective' and 'Always effective' techniques in the 'Wide Tube' and 'Narrow Tube' phases indicates the extent to which chimpanzees altered their behaviour in response to the change in task, and the extent to which they were able to set aside techniques that had been rendered impossible by the change in tube width. An increase in the use of 'Always effective' techniques in the 'Narrow Tube' phase compared with the 'Wide Tube' 
425 phase would indicate a flexible response to the task changes. The 'Narrow Scaffolded' phase is

426 not included in these analyses, in order to exclude the possibility of the scaffolding impacting

427 observed flexibility.

428 [insert Table 2 here]

429 [insert Figure 2 here]

430 The ten individuals that took part in both 'Wide' and 'Narrow' tube phases used 'Always

431 effective' techniques for 58 of 1527 attempts in the 'Wide Tube' phase, increasing to 514 of

4321250 attempts in the 'Narrow Tube' phase. On average, each individual used 'Always effective'

433 techniques for a mean of $8.7 \%(\mathrm{SD}=15.6)$ attempts in the 'Wide Tube' phase, increasing to a

434 mean of $24.2 \% \%(\mathrm{SD}=19.4)$ of attempts in the 'Narrow Tube' phase (see Figure 2). Individual

435 preferences in terms of specific techniques for the 10 individuals that took part in both 'Wide'

436 and 'Narrow' tube phases are shown in Table 3.

437 [insert Table 3 here]

438 The full model (see Table 4) indicates that use of 'Always effective' tool techniques increased 439 significantly in the 'Narrow Tube' phase $(b=2.93, p=.0013)$; in the 'Narrow Tube' phase, 440 chimpanzees were 18.67 (Wald 95\% CI [3.16, 110.47]) times more likely to use an 'Always 441 effective' technique than in the 'Wide Tube' phase. This indicates a significant increase in 442 'Always effective' technique use in the 'Narrow tube' phase, and as we present a binomial 443 GLMM, and each attempt made could only be 'Always effective' or 'Partially effective', an 444 equivalent significant decrease in the use of 'Partially effective' techniques.

445 There was no significant effect of Subgroup $(b=1.57, p=.1631)$ upon the likelihood of an 446 individual using 'Always effective' techniques in either Phase, and no significant interaction 447 between Phase and Subgroup $(b=-1.57, p=.2387)$, indicating that the two Subgroups did not 448 respond significantly differently to the change in Phase. A likelihood ratio test indicated no 449 significant difference between this full model (including the impact of Subgroup and an 450 interaction with Phase) and a reduced model including only Phase $\left(\chi^{2}=1.52, d f=2, p=.4676\right.$; $451 \mathrm{dAIC}=2.4)$, and so we present the full model with all predictors here. 
452 [insert Table 4 here]

453 An additional analysis examining the impact of Session upon the likelihood of individuals using

454 'Always effective' techniques within the 'Narrow Tube' phase found that Session had no

455 significant effect $(b=0.17, p=0.077)$, indicating that individuals did not become more likely to

456 use 'Always effective' techniques as time passed within the 'Narrow Tube' phase.

457 "Narrow Scaffolded" phase

458 Four individuals (Kindia, Edith, Frek, and Pearl) encountered the task with the scaffolded leafy

459 stick solution in place. Of these individuals, only two (Edith and Pearl) put the leaves in their

460 mouths to retrieve the reward. Frek and Kindia instead discarded the branch without retrieving

461 any reward from it. These four individuals (Kindia, Edith, Frek, and Pearl) who encountered the

462 leafy stick solution first-hand did not use the leafy stick solution (dipping the top, leafy part of

463 the branch in, rather than the bare, stick end). It therefore appears that scaffolding the behaviour

464 in this limited manner did not provide sufficient information for these chimpanzees to acquire a

465 novel 'Always effective' solution to the task.

466 Discussion

467 In this study, chimpanzees were found to be capable of responding with some flexibility to a

468 changing task. However, although individuals significantly increased their use of 'Always

469 effective' techniques in the 'Narrow Tube' phase, no individual that made more than one attempt

470 used 'Always effective' techniques for a majority of their attempts. This indicates that

471 chimpanzees' behavioural flexibility was limited by an inability to relinquish the previously

472 successful solutions used in the 'Wide Tube' phase, which continued to comprise at least $50 \%$ of

473 all individuals' attempts. Scaffolding provided to four individuals in an attempt to facilitate use

474 of an 'Always effective' technique in the final 'Narrow Scaffolded' phase did not result in the

475 use of this novel technique by this limited subsample of chimpanzees. Membership of subgroups

476 with differential experiential histories did not have a significant impact upon use of 'Always

477 effective' techniques. The principal issues addressed by the study are discussed in turn below. 
479 Chimpanzees did typically alter their behaviour in response to the change in task, using 480 significantly more 'Always effective' techniques in the 'Narrow Tube' phase. However, no 481 individual that made more than one attempt used 'Always effective' techniques for a majority of 482 their attempts, in contrast with the findings of Manrique et al. (2013) in whose study 483 chimpanzees successfully used an effective solution for the majority of attempts in the 484 appropriate condition. Using a very similar task to the current study, Lehner et al. (2011) found

485 486 that orangutans preferentially used efficient methods when task constraints rendered their previous preferences inefficient. Similarly, Davis et al. (2016) found that most chimpanzees used a novel, efficient solution for the majority of attempts when the efficiency of their previously known solution became very low, though this extent of switching was observed only in groups with a trained demonstrator using the efficient solution or with human demonstrations provided: in 'non-seeded' groups, only one individual switched to the efficient solution, but then used it for the majority of attempts. These comparisons with previous research indicate that whilst chimpanzees in this study showed behavioural flexibility (altered their behaviour), the flexibility was limited in comparison to several previous studies, thus concurring with some other previous studies of chimpanzees (Marshall-Pescini \& Whiten 2008; Hrubesch et al. 2009; Bonnie et al. 2012). The finding also concurs to some extent with experimental studies of wild chimpanzees which have shown that individuals approach novel problems with culturally-informed known behaviours, which may limit the extent to which individuals perceive the affordances of alternative tools (Gruber et al. 2009, 2011). However, evidence from wild observations also shows that chimpanzees are capable of modifying tool behaviour (Yamamoto et al. 2008), and using novel materials to achieve known forms of tool use (Hobaiter et al. 2014), both of which indicate some level of behavioural flexibility in individual wild chimpanzees.

Regarding the 'explore-if-dissatisfied' strategy discussed previously, we found that only one novel technique emerged following the imposition of task constraints (see Table 2), and this technique was used successfully only once. This would seem to indicate that chimpanzees in the current study did not respond to the task changes by exploring the possibility of novel solutions, but rather altered the extent to which they employed known solutions. Three individuals (Louis, Emma and Edith) used a technique that was novel to them in the 'Narrow Tube' phase for a considerable proportion of their attempts (see Table 3). The technique in question (leafy stick dip) was not novel on a group level, and so this may be the result of observational learning (perhaps 
510 indicating a 'copy-if-dissatisfied' strategy). The relatively limited flexibility found in the

511 current study could be due to differences in task demands between the present study and others

512 reporting more flexibility. One possible explanation for the difference in the apparent level of

513 flexibility seen in this study compared with Davis et al. (2016) is the presence in the Davis et al.

514 (2016) study of social information provided by a trained, competent model. The scaffolding in

515 the final 'Narrow Scaffolded' phase aside, chimpanzees were not provided with trained

516 conspecific demonstrators or human demonstrations to offer social information about 'Always

517 effective' techniques. Had the chimpanzees in this study entirely failed to discover 'Always

518 effective' techniques, this lack of experimentally-provided social information would be a

519 plausible explanation for the relatively diminished behavioural flexibility observed. However,

520 the majority of individuals tested used 'Always effective' techniques in the 'Narrow Tube' phase,

521 and indeed had access to social information in the form of others using 'Always effective'

522 techniques, indicating that they were not limited by a lack of knowledge of the existence of such

523 techniques, but rather failed to employ them as frequently as they used 'Partially effective'

524 techniques.

525 As in the current study, apes in Manrique et al. (2013) and Lehner et al. (2011) had to

526 discover more efficient solutions to the provided tasks without additional information from

527 experimenters (though in both Lehner et al., 2011, and the current study, apes were tested in a

528 group context and thus had access to any social information conspecifics provided). Though

529 three individuals did use an 'Always effective' technique that was novel to them in the 'Narrow

530 Tube' phase (Table 3), indicating a capacity for acquisition of novel behaviours via either social

531 or individual learning in response to the change in task, only three of the seven observed

532 solutions (leafy stick dip, leaf squash, and stick drop) were 'Always effective' in the 'Narrow

533 Tube' phase. This limited range of available solutions may have restricted chimpanzees' ability

534 to respond flexibly to the change in task. In comparison, orangutans in the Lehner et al. (2011)

535 study exhibited six effective solutions in 'Restricted Condition 1' (analogous to our 'Narrow

536 Tube' phase) and so had knowledge of a wider range of potential solutions that could be usefully

537 applied to the narrow tube. Comparison of the range of solutions discovered by chimpanzees in

538 the current study with the range of solutions observed by Tonooka, Tomonaga and Matsuzawa

539 (1997) in response to a task similar to the 'Wide Tube' phase of our study may indicate that

540 chimpanzees in our study were somewhat limited in their exploration of the task, with Tonooka 
541 et al. (1997) observing 16 different solutions in comparison to only seven solutions seen in the

542 current study. Although chimpanzees in Tonooka et al. (1997) had access to a wider range of tool

543 materials, which, rather than a lack of exploration, may account for the discrepancy in the

544 number of solutions observed in their study and the current study, there were tool materials

545 available to chimpanzees in our study (namely cloth and wood wool) that were never used in the

546 'Wide Tube' phase, when there were few limitations to the efficacy of solutions.

547 The fact that chimpanzees in our study did not explore the potential of absorbent

548 materials as tool solutions to the task could suggest a role for 'functional fixedness' (Hanus et al.

549 2011) in limiting their exploration of the task. While the chimpanzees are familiar with these

550 absorbent materials, they are primarily used as nesting material. The chimpanzees may therefore

551 have struggled to perceive them as having an alternative use. This does not appear to have

552 constrained the chimpanzees' use of leafy sticks as tools (despite such browse often also being

553 used as nesting material), though their reluctance to use the leaves of these sticks to dip with may

554 also reflect a level of 'functional fixedness', as only the stick portion of such leafy sticks

555 appeared to be considered functional by the chimpanzees.

556 In contrast to the current study, the tasks provided by Manrique et al. (2013) and Davis et 557 al. (2016) did not require the use of tools, and were solvable by hand. Tasks requiring tool use

558 are typically more challenging than those requiring purely manual actions, with the inclusion of

559 tool use more challenging for causal cognition (Seed et al. 2009; Völter \& Call 2014). The

560 necessity of tool use in the latter conditions of this study ('Narrow Tube' phases) may therefore

561 have impeded behavioural flexibility, perhaps by confounding the chimpanzees' comprehension

562 of the efficacy of their solutions, or impeding the acquisition of further 'Always effective'

563 solutions via social or asocial means.

564 The Impact of Scaffolding

565 The limited exposure to scaffolding provided to four chimpanzees in the third and final phase of 566 this study did not lead to the acquisition of novel techniques by any individual. This scaffolding 567 (providing the task with a leafy stick already inserted) aimed to approximate a form of 568 information chimpanzees have access to in the wild - the products and debris of other 569 chimpanzees' tool use, hypothesised to be a facilitator of learning local techniques by Tennie et 570 al. (2009) (e.g. “...nut crackers and termite fishers leave their tools and detritus behind, and in 
571 the right place, which makes the learning of their offspring and others much easier”, pp.2406).

572 This information in the form of residual, enduring artefacts has been argued to facilitate technical 573 activities in New Caledonian crows (Holzaider, Hunt \& Gray 2009), Japanese macaques (Lecca,

574 Gunst \& Hoffman 2010) and bearded capuchins and chimpanzees (Fragaszy et al. 2013),

575 although we are not aware of any direct experimental evidence for such hypothesised effects.

576 Although we are cautious in our interpretation of our results regarding scaffolding, given

577 that only four individuals had very limited interaction with the scaffolding, more extensive

578 efforts to provide this kind of information in experimental settings have often proven

579 unsuccessful in encouraging the invention of novel behaviours. Wild bearded capuchin monkeys

580 from a population that does not habitually use probing tools, when presented with a dipping task

581 with stick probes already inserted, never acquired probing behaviour (Cardoso \& Ottoni 2016).

582 Using a similar task to the current study, Gruber et al. (2009) found that two communities of

583 wild chimpanzees had distinct tool use preferences when extracting honey from a cavity, with

584 one community solving the task using a stick tool while the other solved it using their hands or

585 leaf sponges. Subsequently providing this task to the hand/leaf-sponge community with a stick

586 tool already inserted failed to elicit stick tool use (Gruber et al. 2011). The authors interpret this

587 finding as evidence of "cultural bias" towards existing traditions of tool use, which constrains

588 how individuals perceive and evaluate the affordances of their environment (Gruber et al. 2011),

589 but it may also be that presenting chimpanzees with scaffolded solutions in this manner (at least

590 when numbers of such presentations are limited, with the majority of chimpanzees in Gruber et

591 al.'s study receiving only one or two exposures) simply provides insufficient information to elicit

592 acquisition of novel tool use behaviours. Gruber et al. (2011) argue that such limited exposure

593 may be ecologically valid, and tests the hypothesis that novel tool behaviours will emerge in

594 direct response to favourable affordances (a situation that highlights the physical properties of

595 potential tool materials and indicates the potential relationship between tool and goal), but the

596 possibility remains that such limited exposure to scaffolding is insufficient to impact behaviour.

597 Similarly, experiments incorporating 'ghost conditions', in which the operational

598 affordances of a task are demonstrated without the presence of a demonstrator individual (e.g.

599 task components are moved by fine fishing line or similar), perhaps also corroborates the

600 insufficiency of such scaffolding. Hopper et al. $(2007,2015)$ found that demonstration of the

601 workings of a 'Panpipes' apparatus in such a ghost condition did not lead chimpanzees to 
602 discover the solution to the task. The efficacy of such ghost demonstrations may be affected by

603 the complexity of the task (Hopper et al. 2008), with complex tool use perhaps proving more 604 challenging to learn via such demonstrations. The information provided by these scaffolded 605 conditions (as the current study, and Gruber et al., 2011) can also be argued to be even more 606 impoverished than that provided by ghost conditions as it does not incorporate movement, and so 607 may not demonstrate the affordances of a task to the same extent as moving displays.

608 Providing further groups of chimpanzees with scaffolding approximating the artefacts of 609 other's tool use they might encounter in the wild, perhaps in the context of novel tool use tasks 610 for which no pre-existing cultural biases are likely to exist, could shed further light on the ability 611 of chimpanzees to learn novel behaviours from this sort of information, and indicate whether 612 chimpanzees are limited by cultural biases, by the relative poverty of the information provided 613 by scaffolding, or simply require more extensive exposure to scaffolding in order to acquire 614 novel behaviours.

615 Subgroup differences

616 Subgroup membership was found to have no significant effect on the likelihood of individuals 617 using 'Always effective' techniques in either the 'Wide' or 'Narrow' tube phase, with both 618 groups increasing their use of 'Always effective' techniques in the 'Narrow' tube phases.

619 Previous research has found that chimpanzees reared in captivity perform more poorly than wild620 born chimpanzees in tool-use tasks. Morimura and Mori (2010) found that captive-reared 621 chimpanzees were less likely to succeed than wild-born chimpanzees on a tool use task involving 622 retrieving juice from a bottle using a provided stick tool. Similarly, Brent et al. (1995) found that 623 both captive mother-reared and captive nursery-reared chimpanzees were less likely to succeed 624 than wild-born chimpanzees (in captivity) on a reaching tool task. Rearing history has also been 625 found to have an impact on nest-building, with wild-born chimpanzees in captivity spending 626 more time constructing and using nests than captive-born chimpanzees, and using more complex 627 techniques in their nest building, while within the captive-born chimpanzee sample, mother628 reared individuals spent more time than nursery-born individuals making and using nests 629 (Videan 2006). Some research indicates that housing can influence performance on cognitive 630 tests, with Vlamings, Hare and Call (2010) finding that sanctuary-housed chimpanzees and 631 bonobos outperform zoo-housed apes in a detour-reaching task testing inhibitory control. 632 However, Wobber and Hare (2011), using a subset of the Primate Cognition Test Battery 
633 (Herrmann et al. 2007), found no impact of housing (zoo vs sanctuary) on the performance of

634 mother-reared chimpanzees on tests of social or physical cognition, but did find that mother-

635 reared individuals (regardless of housing) outperformed orphaned individuals on physical

636 cognition tests - this effect was due to improved performance on a tool properties task in which

637 individuals chose between a functional and non-functional tool. Similarly, Herrmann, Wobber

638 and Call (2008) found no difference in performance between zoo- and sanctuary-housed

639 chimpanzees and orangutans on a tool functionality choice task.

640 We did not find evidence of subgroup differences in the current study, though the

641 subgroups compared here were based upon the chimpanzees' housing history rather than their

642 rearing. Although only the Beekse Bergen group includes individuals hand-reared by humans,

643 and this is the most common rearing history for this subgroup (five of nine individuals), both

644 groups include both wild- and captive-born mother-reared individuals. Sample size precluded

645 any analysis based upon rearing history (with only three individuals categorised as wild-born,

646 only two of which participated in both the 'Wide' and 'Narrow Tube' phases of the task), and

647 given the difference in origin of the two groups (laboratory vs. zoo) it was thought a subgroup

648 analysis might capture any differences in performance related to early life experiences. It may be

649 that, between captive environments housing chimpanzees with mixed rearing histories, there is

650 little difference in performance to be found using artificial foraging tasks such as the current task.

651 However, further groups of chimpanzees in different captive facilities would have to be provided

652 with the task in order to test this. Examination of the impact of both housing and rearing histories

653 upon chimpanzee performance in experimental tasks is essential for a full understanding of

654 chimpanzee cognition (Boesch 2007).

655 Limitations to the current study

656 Although some previous studies of chimpanzee and great ape behavioural flexibility have tested

657 individuals in isolation (Marshall-Pescini \& Whiten 2008; Manrique \& Call 2013, 2015), and

658 this approach allows for control over chimpanzees' access to social information about the task

659 and the avoidance of issues around task monopolization by dominant individuals, we note that

660 many previous studies have presented tasks in a group context (Gruber et al. 2009, 2011; Lehner

661 et al. 2011; Davis et al. 2016). This may be due to the constraints of conducting research at

662 captive facilities (or, in the case of Gruber et al., 2009, 2011, in the wild), or be a methodological 
663 choice in order to explore the social learning abilities of subjects (Davis et al. 2016). The open

664 presentation of the task in the current study to the group of chimpanzees prevents us from

665 making claims regarding the process by which novel behaviours emerged and spread in

666 individuals during the trials (whether this occurred via social learning or innovation). However,

667 following Lehner et al.'s (2011) definition of behavioural flexibility, as the continued acquisition

668 of novel techniques via either social or asocial learning, group testing in this manner allows

669 flexibility to be demonstrated through either form of learning.

670 This study focused upon a community of chimpanzees with particular life histories

671 (primarily captive born) and was also limited to adult chimpanzees, with no infants or juveniles

672 in the population. Previous research has shown that behavioural flexibility (referred to as

673 'cognitive flexibility' by Manrique and Call, 2015, and measured by a simple reversal task)

674 follows a U-shaped curve in great apes, with optimum performance in terms of error avoidance

675 appearing to occur between seven and 27 years of age (Manrique \& Call 2015), and so the age of

676 some individuals in the current study may have impaired their performance (with five of the ten

677 individuals that provided data in both the 'Wide' and 'Narrow Tube' phases being over 27 years

678 old). Position in the dominance hierarchy could also impact performance on an artificial foraging

679 task such as that of the current study. Low ranking individuals may be more likely to innovate

680 novel behaviours (Reader \& Laland 2001), and in a group context, dominance may impact the

681 likelihood of others acquiring a behaviour from an individual via social learning (Horner et al.

682 2010; Kendal et al. 2015, though see also Watson et al. 2017). With a sample of only ten

683 individuals providing data across the 'Wide' and 'Narrow Tube' phases, analyses of age, rearing

684 history, and rank effects were not possible in the current study, but these previous findings

685 demonstrate the importance of considering the impact of such factors upon task performance

686 when possible. Future work would benefit from expanding the sample to include a more diverse

687 range of ages and rearing histories, in sufficient numbers to enable analysis of the potential

688 effects of these and other factors upon participation and performance in artificial foraging tasks.

$689 \underline{\text { Conclusions }}$

690 Chimpanzees in this study did respond flexibly to a changing task, increasing their use of

691 'Always effective' techniques when task alterations rendered previously used solutions

692 unrewarding. However, no individual altered their behaviour to the extent of using 'Always 
693 effective' solutions for a majority of attempts, indicating relatively limited behavioural flexibility

694 in comparison with some previous research (Lehner et al. 2011; Manrique et al. 2013; Davis et al. 695 2016) and a tendency to continue to use previously rewarded behaviours. The relatively limited 696 flexibility observed here may be due to the complexity of the task, which required tool use. A 697 subset of chimpanzees did not acquire a more effective, novel, tool technique when provided 698 with a single instance of scaffolding towards the solution.

699 Research on behavioural flexibility in chimpanzees, both in the wild and in captivity, 700 using a variety of artificial foraging tasks, continues to provide divergent results, with some 701 studies indicating strong conservatism, while others find an ability to relinquish known solutions 702 in favour of more rewarding or efficient techniques. Further work must been done to investigate 703 what factors limit or encourage flexibility in great apes, with promising avenues for further study 704 being the relative complexity of the task (for example, the requirement for tool use versus tasks 705 which can be solved by hand), the disparity in efficacy between known and alternative 706 techniques (whether the established solutions become entirely unrewarded or simply less 707 efficient or rewarding than an alternative) and investigation of individual characteristics such as 708 age or rank which may promote or constrain behavioural flexibility. 
713

714

715

716

717

718

719

720

721

722

723

724

725

726

727

728

729

730

731

732

733

734

735

736

737

738

739

740

741

742

743

744

745

746

$\underline{\text { References }}$

Audet JN, Lefebvre L. 2017. What's flexible in behavioral flexibility? Behavioral Ecology, 28:943-947. doi: 10.1093/beheco/arx007

Auersperg AMI, von Bayern AMP, Gajdon GK, Huber L, Kacelnik A. 2011. Flexibility in problem solving and tool use of kea and New Caledonian crows in a multi access box paradigm. PLoS ONE 6:e20231. doi:10.1371/journal.pone.0020231

Bates D, Maechler M, Bolker B. 2012. lme4: Linear mixed-effects models using S4 classes. R package version 0.999999-0. Retrieved from http://CRAN.R-project.org/package=lme4

Benus RF, Den Daas S, Koolhaas JM, Van Oortmerssen GA. 1990. Routine formation and flexibility in social and non-social behaviour of aggressive and non-aggressive male mice. Behaviour, 112:176-193. doi: 10.1163/156853990X00185

Bloomsmith MA, Baker KC, Ross SR, Lambeth SP. 2006. Early rearing conditions and captive chimpanzee behavior: some surprising findings. In: Sackett GP, Ruppenthal GC, Elias K, eds. Nursery rearing of nonhuman primates in the 21st century. Springer: New York, 289-312.

Boesch C. 2007. What makes us human (Homo sapiens)? The challenge of cognitive crossspecies comparison. Journal of Comparative Psychology, 121:227-240. doi: 10.1037/0735-7036.121.3.227

Bolhuis JE, Schouten WG, de Leeuw JA, Schrama JW, Wiegant VM. 2004. Individual coping characteristics, rearing conditions and behavioural flexibility in pigs. Behavioral Brain Research, 152:351-360. doi: 10.1016/j.bbr.2003.10.024

Bolker BM, Brooks ME, Clark CJ, Geange SW, Poulsen JR, Stevens MH, White JS. 2009. Generalized linear mixed models: a practical guide for ecology and evolution. Trends in Ecology \& Evolution, 24:127-35. doi: 10.1016/j.tree.2008.10.008

Bond AB, Kamil AC, Balda RP. 2007. Serial reversal learning and the evolution of behavioral flexibility in three species of North American corvids (Gymnorhinus cyanocephalus, Nucifraga columbiana, Aphelocoma californica). Journal of Comparative Psychology, 121: 372-379. doi:10.1037/0735-7036.121.4.372

Bonnie KE, Milstein MS, Calcutt SE, Ross SR, Wagner KE, Lonsdorf EV. 2012. Flexibility and persistence of chimpanzee (Pan troglodytes) foraging behaviour in a captive environment. American Journal of Primatology, 74:661-668. doi:10.1002/ajp.22020

Boogert NJ, Monceau K, Lefebvre L. 2010. A field test of behavioural flexibility in Zenaida doves (Zenaida aurita). Behavioural Processes, 85:135-141. doi:10.1016/j.beproc.2010.06.020

Brent L, Bloomsmith MA, Fisher SD. 1995. Factors determining tool-using ability in two captive chimpanzee (Pan troglodytes) colonies. Primates, 36:265-274. doi:10.1007/BF02381352

Brosnan SF, Hopper LM. 2014. Psychological limits on animal innovation. Animal Behavior, 92:325-32. doi: 10.1016/j.anbehav.2014.02.026 
747 Cardoso RM, Ottoni EB. 2016. The effects of tradition on problem solving by two wild

748

749

750

751

752

753

754

755

756

757

758

759

760

761

762

763

764

765

766

767

768

769

770

771

772

773

774

775

776

777

778

779

780

781

782

783

784

785

786

populations of bearded capuchin monkeys in a probing task. Biology Letters, 12:20160604. doi: 0.1098/rsbl.2016.0604

Charbonneau M. 2015. All innovations are equal, but some more than others: (Re)integrating modification processes to the origins of cumulative culture. Biological Theory, 10:322335. doi: 10.1007/s13752-015-0227-x

Chow PKY, Lea SE, Leaver LA. 2016. How practice makes perfect: the role of persistence, flexibility and learning in problem-solving efficiency. Animal Behavior, 112:273-283. doi:10.1016/j.anbehav.2015.11.014

Davis SJ, Vale GL, Schapiro SJ, Lambeth SP, Whiten A. 2016. Foundations of cumulative culture in apes: Improved foraging efficiency through relinquishing and combining witnessed behaviours in chimpanzees (Pan troglodytes). Scientific Reports, 6:35953. doi: $10.1038 /$ srep35953

Dean LG, Vale GL, Laland KN, Flynn E, Kendal RL. 2014. Human cumulative culture: a comparative perspective. Biological Reviews of the Cambridge Philosophical Society, 89:284-301. doi:10.1111/brv.12053

Fragaszy DM, Biro D, Eshchar Y, Humle T, Izar P, Resende B, Visalberghi E. 2013. The fourth dimension of tool use: temporally enduring artefacts aid primate learning to use tools. Philosophical Transactions of the Royal Society B: Biological Sciences, 368:20120410. doi: $10.1098 / \mathrm{rstb} .2012 .0410$

Griffin AS, Guez D. 2014. Innovation and problem solving: A review of common mechanisms. Behavioural Processes, 109:121-134. doi: 10.1016/j.beproc.2014.08.027

Griffin AS, Guez D, Lermite F, Patience M. 2013. Tracking changing environments: Innovators are fast, but not flexible learners. PLoS ONE, 8:e84907. doi:10.1371/journal.pone.0084907

Gruber T. 2016. Great apes do not learn novel tool use easily: Conservatism, functional fixedness, or cultural influence? International Journal of Primatology, 37:296-316. doi: 10.1007/s10764-016-9902-4

Gruber T, Muller MN, Reynolds V, Wrangham RW, Zuberbühler K. 2011. Community-specific evaluation of tool affordances in wild chimpanzees. Scinetific Reports, 1:128. doi: $10.1038 /$ srep00128

Gruber T, Muller MN, Strimling P, Wrangham RW, Zuberbühler K. 2009. Wild chimpanzees rely on cultural knowledge to solve an experimental honey acquisition task. Current Biology, 19:1806-1810. doi: 10.1016/j.cub.2009.08.060

Hanus D, Mendes N, Tennie C, Call J. 2011. Comparing the performances of apes (Gorilla gorilla, Pan troglodytes, Pongo pygmaeus) and human children (Homo sapiens) in the floating peanut task. PLoS ONE, 6:e19555. doi:10.1371/journal.pone.0019555

Herrmann E, Call J, Hernandez-Lloreda MV, Hare B, Tomasello M. 2007. Humans have evolved specialized skills of social cognition: The cultural intelligence hypothesis. Science, 317:1360. doi: 10.1126/science. 1146282

Peer] reviewing PDF | (2017:09:20869:3:0:NEW 22 Jan 2018) 
787

788

789

790

791

792

793

794

795

796

797

798

799

800

801

802

803

804

805

806

807

808

809

810

811

812

813

814

815

816

817

818

819

820

821

822

823

824

825

Hobaiter C, Poisot T, Zuberbühler K, Hoppitt W, Gruber T. 2014. Social network analysis shows direct evidence for social transmission of tool use in wild chimpanzees. PLoS Biology, 12: e1001960. doi: 10.1371/journal.pbio. 1001960

Holzhaider JC, Hunt GR, Gray RD. 2010. The development of pandanus tool manufacture in wild New Caledonian crows. Behaviour, 147:553-586. doi: 10.1163/000579510X12629536366284

Hopper LM, Spiteri A, Lambeth SP, Schapiro SJ, Horner V, Whiten A. 2007. Experimental studies of traditions and underlying transmission processes in chimpanzees. Animal Behavior, 73:1021-1032. doi: 10.1016/j.anbehav.2006.07.016

Hopper LM, Lambeth SP, Schapiro SJ, Whiten A. 2008. Observational learning in chimpanzees and children studied through 'ghost' conditions. Proceedings of the Royal Society B: Biological Sciences, 275:835-840. doi: 10.1098/rspb.2007.1542

Hopper LM, Lambeth SP, Schapiro SJ, Whiten A. 2015. The importance of witnessed agency in chimpanzee social learning of tool use. Behavioral Processes, 112:120-129. doi: 10.1016/j.beproc.2014.10.009

Horner V, Proctor D, Bonnie KE, Whiten A, de Waal FB. 2010. Prestige affects cultural learning in chimpanzees. PloS ONE 5:e10625. doi:10.1371/journal.pone.0010625

Hrubesch C, Preuschoft S, van Schaik C. 2009. Skill mastery inhibits adoption of observed alternative solutions among chimpanzees (Pan troglodytes). Animal Cognition, 12:209216. doi: 10.1007/s10071-008-0183-y

Jaeger TF. 2008. Categorical data analysis: Away from ANOVAs (transformation or not) and towards logit mixed models. Journal of Memory and Language, 59:434-446. doi:10.1016/j.jml.2007.11.007

Kendal R, Hopper LM, Whiten A, Brosnan SF, Lambeth SP, Schapiro SJ, Hoppitt W. 2015. Chimpanzees copy dominant and knowledgeable individuals: implications for cultural diversity. Evolution and Human Behavior, 36:65-72. doi: 10.1016/j.evolhumbehav.2014.09.002

Klump BC, Sugasawa S, St Clair JJH, Rutz C. 2015. Hook tool manufacture in New Caledonian crows: Behavioural variation and the influence of raw materials. BMC Biology, 13:97. doi:10.1186/s12915-015-0204-7

Laland, KN. 2004. Social learning strategies. Learning \& Behavior, 32:4-14. doi:10.3758/BF03196002

Laland KN, Hoppitt W. 2003. Do animals have culture? Evolutionary Anthropology, 12:150-159. doi:10.1002/evan.10111

Leca JB, Gunst N, Huffman MA. 2010. Indirect social influence in the maintenance of the stonehandling tradition in Japanese macaques, Macaca fuscata. Animal Behavior, 79:117-126. doi: 10.1016/j.anbehav.2009.09.035

Lefebvre L, Whittle P, Lascaris E, Finkelstein A. 1997. Feeding innovations and forebrain size in birds. Animal Behavior, 53:549-560. doi: 10.1006/anbe.1996.0330 
826 Lefebvre L, Reader SM, Sol D. 2004. Brains, innovations and evolution in birds and primates.

827

828

829

830

831

832

833

834

835

836

837

838

839

840

841

842

843

844

845

846

847

848

849

850

851

852

853

854

855

856

857

858

859

860

861

862

863

864 Brain, Behavior and Evolution, 63:233-246. doi: 10.1159/000076784

Lehner SR, Burkart JM, van Schaik CP. 2011. Can captive orangutans (Pongo pygmaeus abelii) be coaxed into cumulative build-up of techniques? Journal of Comparative Psychology, 125: 446-455. doi: 10.1037/a0024413

Lewis HM, Laland KN. 2012. Transmission fidelity is the key to the build-up of cumulative culture. Philosophical Transactions of the Royal Society B: Biological Sciences, 367:2171-80. doi: 10.1098/rstb.2012.0119

Liu Y, Day LB, Summers K, Burmeister SS. 2016. Learning to learn: Advanced behavioural flexibility in a poison frog. Animal Behavior, 111:167-172. doi:10.1016/j.anbehav.2015.10.018

Logan CJ. 2016. How far will a behaviourally flexible invasive bird go to innovate? Royal Society Open Science, 3:160247. doi: 10.1098/rsos.160247

Manrique HM, Call J. 2015. Age-dependent cognitive inflexibility in great apes. Animal Behavior, 102:1-6. doi: 10.1016/j.anbehav.2015.01.002

Manrique HM, Völter CJ, Call J. 2013. Repeated innovation in great apes. Animal Behavior, 85:195-202. doi: 10.1016/j.anbehav.2012.10.026

Marshall-Pescini S, Whiten A. 2008. Chimpanzees (Pan troglodytes) and the question of cumulative culture: an experimental approach. Animal Cognition, 11:449-456. doi:10.1007/s10071-007-0135-y

Massen JJ, Antonides A, Arnold AM, Bionda T, Koski SE. 2013. A behavioral view on chimpanzee personality: Exploration tendency, persistence, boldness, and tool - orientation measured with group experiments. American Journal of Primatology, 75:947-58. doi: 10.1002/ajp.22159

Morimura N, Mori Y. 2010. Effects of early rearing conditions on problem-solving skill in captive male chimpanzees (Pan troglodytes). American Journal of Primatology, 72:626633. doi:10.1002/ajp.20819

Mulcahy N J, Call J. 2006. How great apes perform on a modified trap-tube task. Animal Cognition, 9:193-199. doi: 10.1007/s10071-006-0019-6

Povinelli DJ. 2000. Folk physics for apes: a chimpanzee's theory of how the mind works. Oxford University Press, Oxford

Price EE, Lambeth SP, Schapiro SJ, Whiten A. 2009. A potent effect of observational learning on chimpanzee tool construction. Proceedings of the Royal Society B: Biological Sciences, 276:3377-3383 doi: 10.1098/rspb.2009.0640

R Core Team. 2015. R: A language and environment for statistical computing. R Foundation for Statistical Computing, Vienna, Austria. URL https:/www.R-project.org/.

Reader SM, Laland KN. 2001. Primate innovation: sex, age and social rank differences. International Journal of Primatology, 22:787-805. doi: 10.1023/A:1012069500899

Reader SM, Laland KN (Eds). 2003. Animal innovation. Oxford: Oxford University Press. 
865

866

867

868

869

870

871

872

873

874

875

876

877

878

879

880

881

882

883

884

885

886

887

888

889

890

891

892

893

894

895

896

897

898

899

900

901

902
Richter JN, Hochner B, Kuba MJ. 2016. Pull or push? Octopuses solve a puzzle problem. PLoS ONE, 11:e0152048. doi:10.1371/journal.pone.0152048

RStudio Team. 2015. RStudio: Integrated Development for R. RStudio, Inc., Boston, MA URL http://www.rstudio.com/.

Rutz C, Sugasawa S, van der Wal JEM, Klump BC, St Clair JJH. 2016. Tool bending in New Caledonian crows. Royal Society Open Science, 3:160439. doi:0.1098/rsos.160439

Sanz C, Call J, Morgan D. 2009. Design complexity in termite-fishing tools of chimpanzees (Pan troglodytes). Biology Letters, 5:293-296. doi: 10.1098/rsbl.2008.0786

Schel AM, Rawlings B, Claidiere N, Wilke C, Wathan J, Richardson J, Pearson S, Herrelko ES, Whiten A, Slocombe K. 2012. Network analysis of social changes in a captive chimpanzee community following the successful integration of two adult groups. American Journal of Primatology, 75:254-266. doi:10.1002/ajp.22101

Schielzeth H, Forstmeier W. 2009. Conclusions beyond support: overconfident estimates in mixed models. Behavioral Ecology, 20:416-420. doi:10.1093/beheco/arn145

Seed AM, Call J, Emery NJ, Clayton NS. 2009. Chimpanzees solve the trap problem when the confound of tool-use is removed. Journal of Experimental Psychology: Animal Behavioral Processes, 35:23-34. doi:10.1037/a0012925

Silva FJ, Page DM, Silva KM. 2005. Methodological-conceptual problems in the study of chimpanzees' folk physics: how studies with adult humans can help. Learning \& Behavior, 33:47-58. doi: 10.3758/BF03196049

Sol D, Timmermans S, Lefebvre L. 2002. Behavioural flexibility and invasion success in birds. Animal Behavior, 63:495-502. doi: 10.1006/anbe.2001.1953

St Clair JJH, Klump BC, van der Wal JEM, Sugasawa S, Rutz C. 2016. Strong between-site variation in New Caledonian crows' use of hook-tool-making materials. Biological Journal of the Linnean Society, 118:226-232. doi:10.1111/bij.12757

Templeton JJ, Kamil AC, Balda RP. 1999. Sociality and social learning in two species of corvids: The pinyon jay (Gymnorhinus cyancephalus) and the Clark's nutcracker (Nucifraga columbiana). Journal of Comparative Psychology, 113:450-455. doi: 10.1037/07357036.113.4.450

Tennie C, Call J, Tomasello M. 2009. Ratcheting up the ratchet: On the evolution of cumulative culture. Philosophical Transactions of the Royal Society B: Biological Sciences, 364:2405-2415. doi:10.1098/rstb.2009.0052

Tomasello, M. 1994. The question of chimpanzee culture. In: Wrangham R, McGrew WC, De Waal FB, Heltne PG. (Eds.) Chimpanzee cultures. Harvard University Press, Cambridge, Massachusetts, pp 301-319

Tonooka R, Tomonaga M, Matsuzawa T. 1997. Acquisition and transmission of tool making and use for drinking juice in a group of captive chimpanzees (Pan troglodytes). Japanese Psychological Research, 39:253-265. doi:10.1111/1468-5884.00058 
903 Vale GL, Davis SJ, Lambeth SP, Schapiro SJ, Whiten A. 2017. Acquisition of a socially learned 904 tool use sequence in chimpanzees: Implications for cumulative culture. Evolution and $905 \quad H u m a n$ Behavior, 38:635-644 doi:10.1016/j.evolhumbehav.2017.04.007

906 Videan EN. 2006. Bed-building in captive chimpanzees (Pan troglodytes): The importance of 907 early rearing. American Journal of Primatology, 68:745-751. doi:10.1002/ajp.20265

908 Vlamings PHJM, Hare B, Call J. 2010. Reaching around barriers: the performance of the great $909 \quad$ apes and 3 - 5-year-old children. Animal Cognition, 13:273-285. doi:10.1007/s10071$910 \quad 009-0265-5$

911 Völter CJ, Call J. 2014. The cognitive underpinnings of flexible tool use in great apes. Journal of 912 Experimental Psychology: Animal Learning and Cognition, 40:287-302. doi:10.1037/xan0000025

Wallace EK, Altschul D, Körfer K, Benti B, Kaeser A, Lambeth S, Waller BM, Slocombe KE. 2017. Is music enriching for group-housed captive chimpanzees (Pan troglodytes)? PLoS ONE, 12:e0172672. doi:10.1371/journal.pone.0172672

Watson SK, Townsend SW, Schel AM, Wilke C, Wallace EK, Cheng L, West V, Slocombe K. 2015. Vocal learning in the functionally referential food grunts of chimpanzees. Current Biology, 25:495-499. doi:10.1016/j.cub.2014.12.032

Watson SK, Reamer LA, Mareno MC, Vale G, Harrison RA, Lambeth SP, Schapiro SJ, Whiten A. 2017. Socially transmitted diffusion of a novel behavior from subordinate chimpanzees. American Journal of Primatology, 79:e22642. doi:10.1002/ajp.22642

Whiten, A. 2017. Social learning and culture in child and chimpanzee. Annual Review of Psychology, 68:129-154 doi:10.1146/annurev-psych-010416-044108

Whiten A, Horner V, de Waal FBM. 2005. Conformity to cultural norms of tool use in chimpanzees. Nature, 437:737-740. doi: 10.1038/nature04047

Wright TF, Eberhard JR, Hobson EA, Avery ML, Russello MA. 2010. Behavioral flexibility and species invasions: the adaptive flexibility hypothesis. Ethology, Ecology \& Evolution, 22:393-404. doi: 10.1080/03949370.2010.505580

Wobber V, Hare B. 2011. Psychological health of orphan bonobos and chimpanzees in African sanctuaries. PLoS ONE, 6:e17147 doi: 10.1371/journal.pone.0017147

Yamamoto S, Yamakoshi G, Humle T, Matsuzawa T. 2008. Invention and modification of a new tool use behaviour: Ant-fishing in trees by a wild chimpanzee (Pan troglodytes verus) at Bossou, Guinea. American Journal of Primatology, 70:699-702. doi: 10.1002/ajp.20544 


\section{Figure 1}

Image of the apparatus as presented to chimpanzees.

The 'wide' tube as presented to the chimpanzees within the research pod. The 'narrow' tube was presented in the same manner. The height of both tubes was $30 \mathrm{~cm}$, with the 'wide' tube diameter measuring $10 \mathrm{~cm}$ and the 'narrow' tube diameter measuring $5 \mathrm{~cm}$.

*Note: Auto Gamma Correction was used for the image. This only affects the reviewing manuscript. See original source image if needed for review. 


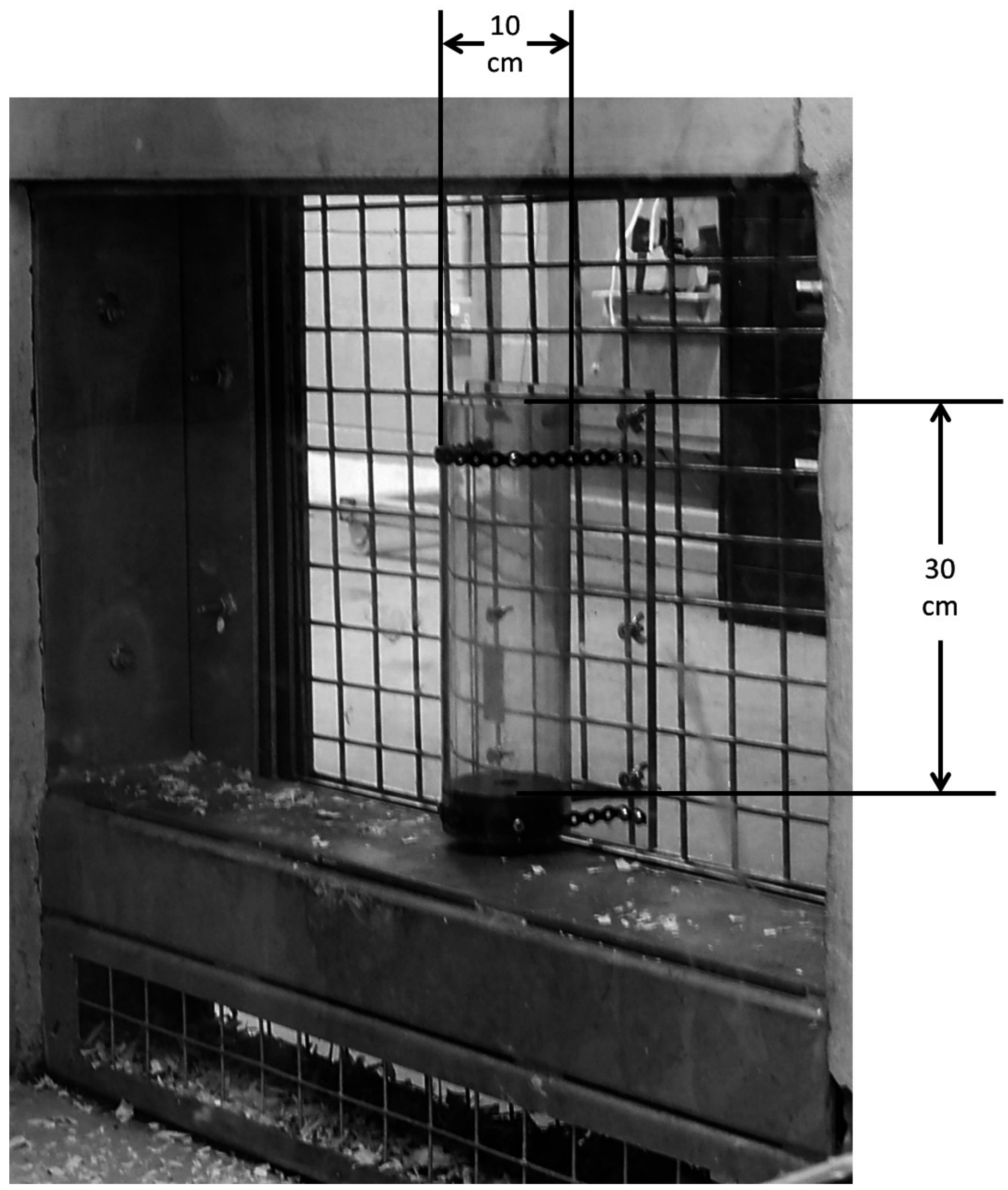


Figure 2

Proportion of attempts made using 'Always effective' techniques in 'Wide' vs 'Narrow' tube phases by the ten chimpanzees that took part in both phases.

Individual chimpanzees' proportions are indicated by dots. Coloured horizontal bars show each subgroup's mean proportion. Black horizontal bars show overall mean proportion. Note that in the 'Narrow Tube' phase, the subgroup means and overall mean are the same.

$1.00-$

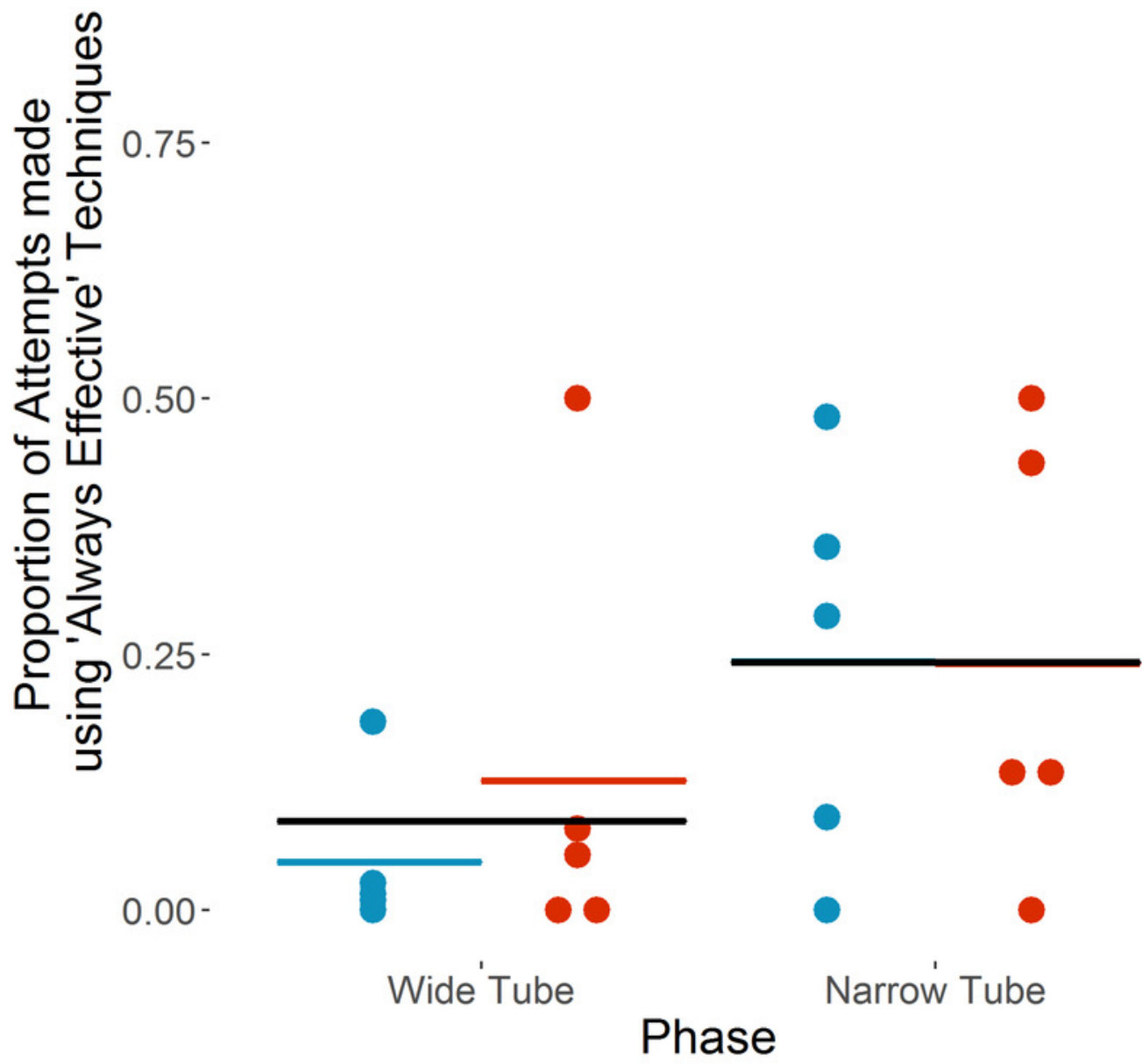




\section{Table $\mathbf{1}$ (on next page)}

Demographic and rearing information of the chimpanzees with level of participation in current study 
Table 1.

Demographic and Rearing Information of the Chimpanzees with Level of Participation in Current Study.

\begin{tabular}{|c|c|c|c|c|c|c|c|c|}
\hline \multirow[t]{2}{*}{ Subgroup } & \multirow[t]{2}{*}{ Individual } & \multirow[t]{2}{*}{ Sex } & \multirow{2}{*}{$\begin{array}{r}\text { Year of } \\
\text { birth } \\
\text { (age at } \\
\text { time of } \\
\text { testing) }\end{array}$} & \multirow{2}{*}{$\begin{array}{l}\text { Origin } \\
\text { (wild } \\
\text { or } \\
\text { captive } \\
\text { born) }\end{array}$} & \multirow[t]{2}{*}{ Rearing } & \multicolumn{3}{|c|}{$\begin{array}{l}\text { Participation in current } \\
\text { study (number of attempts) }\end{array}$} \\
\hline & & & & & & $\begin{array}{l}\text { Wide } \\
\text { Tube }\end{array}$ & $\begin{array}{r}\text { Narrow } \\
\text { Tube }\end{array}$ & $\begin{array}{r}\text { Narrow } \\
\text { Scaffolded }\end{array}$ \\
\hline \multirow[t]{9}{*}{ Edinburgh } & Qafzeh & $\mathrm{M}$ & $\begin{array}{r}1992 \\
(22)\end{array}$ & Captive & Mother & 0 & 1 & 0 \\
\hline & Kindia & M & $\begin{array}{r}1997 \\
(17)\end{array}$ & Captive & Mother & 14 & 4 & 1 \\
\hline & Liberius & M & $\begin{array}{r}1999 \\
(15)\end{array}$ & Captive & Mother & 1 & 0 & 1 \\
\hline & David & M & $\begin{array}{r}1975 \\
(39)\end{array}$ & Captive & Mother & 4 & 0 & 0 \\
\hline & Louis & $\mathrm{M}$ & $\begin{array}{r}1976 \\
(38)\end{array}$ & Wild & & 6 & 15 & 0 \\
\hline & Lucy & $\mathrm{F}$ & $\begin{array}{r}1976 \\
(38)\end{array}$ & Captive & Mother & 11 & 2 & 0 \\
\hline & Kilimi & $\mathrm{F}$ & $\begin{array}{r}1993 \\
(21)\end{array}$ & Captive & Mother & 175 & 103 & 87 \\
\hline & Cindy & $\mathrm{F}$ & $\begin{array}{r}1964 \\
(50)\end{array}$ & Wild & & 0 & 0 & 10 \\
\hline & Emma & $\mathrm{F}$ & $\begin{array}{r}1981 \\
(33)\end{array}$ & Captive & Mother & 37 & 59 & 0 \\
\hline \multirow[t]{9}{*}{$\begin{array}{l}\text { Beekse } \\
\text { Bergen }\end{array}$} & Paul & M & $\begin{array}{r}1993 \\
(21)\end{array}$ & Captive & $\begin{array}{l}\text { Hand- } \\
\text { raised }\end{array}$ & 0 & 0 & 0 \\
\hline & Pearl & F & $\begin{array}{r}1969 \\
(45)\end{array}$ & Wild & & 311 & 214 & 40 \\
\hline & Sofie & $\mathrm{F}$ & $\begin{array}{r}1981 \\
(33)\end{array}$ & Captive & $\begin{array}{l}\text { Hand- } \\
\text { raised }\end{array}$ & 3 & 0 & 2 \\
\hline & Lianne & $\mathrm{F}$ & $\begin{array}{r}1989 \\
(25)\end{array}$ & Captive & Mother & 0 & 0 & 0 \\
\hline & Heleen & $\mathrm{F}$ & $\begin{array}{r}1991 \\
(23)\end{array}$ & Captive & Mother & 4 & 0 & 0 \\
\hline & Edith & $\mathrm{F}$ & $\begin{array}{r}1996 \\
(18)\end{array}$ & Captive & Mother & 385 & 741 & 73 \\
\hline & Eva & $\mathrm{F}$ & $\begin{array}{r}1980 \\
(34)\end{array}$ & Captive & $\begin{array}{l}\text { Hand- } \\
\text { raised }\end{array}$ & 311 & 21 & 4 \\
\hline & Frek & M & $\begin{array}{r}1993 \\
(21)\end{array}$ & Captive & $\begin{array}{l}\text { Hand- } \\
\text { raised }\end{array}$ & 125 & 80 & 11 \\
\hline & Rene & M & $\begin{array}{r}1993 \\
(21)\end{array}$ & Captive & $\begin{array}{l}\text { Hand- } \\
\text { raised }\end{array}$ & 152 & 11 & 3 \\
\hline
\end{tabular}


Note: In addition to their own attempts, all individuals with the exception of Cindy and Liberius were present in the research pods during another individual's attempt on at least one occasion. 


\section{Table 2 (on next page)}

Techniques successfully used to solve the task

Note: Dashed horizontal line indicates transition between the 'Wide Tube' and 'Narrow Tube' phases. 
Table 2.

Techniques successfully used to solve the task

\begin{tabular}{|c|c|c|c|c|c|}
\hline Technique & Description & $\begin{array}{l}\text { Latency to first } \\
\text { successful use } \\
\text { from start of first } \\
\text { testing session }\end{array}$ & $\begin{array}{l}\text { Effective in 'Wide } \\
\text { Tube' phase? }\end{array}$ & $\begin{array}{l}\text { Maintained } \\
\text { efficacy in } \\
\text { 'Narrow Tube' } \\
\text { phase? }\end{array}$ & $\begin{array}{l}\text { Effective in all } \\
\text { phases? }\end{array}$ \\
\hline Hand dip & Dip hand directly into juice & $00: 00: 35$ & Yes & No & Partially effective \\
\hline Stick dip & Stick is dipped directly into the juice & $00: 00: 43$ & Yes & No & Partially effective \\
\hline Stick retrieve & Stick already in tube removed with hand & $00: 23: 38$ & Yes & No & Partially effective \\
\hline Stick drop & Drop stick into tube then retrieve by hand & $00: 21: 16$ & Yes & Yes & Always effective \\
\hline \multirow[t]{4}{*}{ Leafy stick dip } & Dip stick end of a leafy branch directly into the juice & $02: 47: 23$ & Yes & Yes & Always effective \\
\hline & (this differs from 'stick dip' only in the material) This & & & & \\
\hline & technique remained Effective in Narrow tube phases & & & & \\
\hline & due to the flexibility of the leafy sticks in comparison & & & & \\
\hline \multirow[t]{4}{*}{ Leaf squash } & $\begin{array}{l}\text { to standard sticks } \\
\text { A leafy branch is inserted stick first, and the leaves are }\end{array}$ & $05: 56: 26$ & Yes & Yes & Always effective \\
\hline & then forced down into the tube into contact with the & & & & \\
\hline & juice & & & & \\
\hline & (Note: this technique was used successfully only once) & & & & \\
\hline \multirow{3}{*}{$\begin{array}{l}\text { Wood wool } \\
\text { retrieve }\end{array}$} & Wood wool pushed part way into tube by a previous & 11:43:12 & Yes & No & Partially effective \\
\hline & individual removed with hand & & & & \\
\hline & (Note: this technique was used successfully only once) & & & & \\
\hline
\end{tabular}

Note: Dashed horizontal line indicates transition between the 'Wide Tube' and 'Narrow Tube' phases. The 'Narrow Tube'.phase began at 8:17:44 (the start of the $11^{\text {th }}$ experimental session). 


\section{Table 3(on next page)}

The two most frequently used techniques of individuals that made attempts in both the 'Wide' and 'Narrow' tube phases.

'Always effective' techniques are shown in bold. An asterisk indicates that an individual used a technique for the first time within the 'Narrow' Tube phase (ie. that the technique was novel to them). Note: Percentages are rounded. 
1 Table 3.

2 The two most frequently used techniques of individuals that made attempts in both the 'Wide'

3 and 'Narrow' tube phases. 'Always effective' techniques are shown in bold. An asterisk indicates

4 that an individual used a technique for the first time within the 'Narrow' Tube phase (ie. that the

5 technique was novel to them).

\begin{tabular}{|c|c|c|c|c|c|}
\hline \multirow[t]{2}{*}{ Subgroup } & \multirow[t]{2}{*}{ Individual } & \multicolumn{2}{|c|}{ 'Wide Tube' phase } & \multicolumn{2}{|c|}{ 'Narrow Tube' phase } \\
\hline & & $\begin{array}{l}1^{\text {st }} \text { preferred } \\
\text { technique (no. } \\
\text { of attempts; \% } \\
\text { of attempts) }\end{array}$ & $\begin{array}{l}2^{\text {nd }} \text { preferred } \\
\text { technique (no. of } \\
\text { attempts; \% of } \\
\text { attempts) }\end{array}$ & $\begin{array}{l}1^{\text {st }} \text { preferred } \\
\text { technique (no. } \\
\text { of attempts; \% } \\
\text { of attempts) }\end{array}$ & $\begin{array}{l}2^{\text {nd }} \text { preferred } \\
\text { technique (no. of } \\
\text { attempts; } \% \text { of } \\
\text { attempts) }\end{array}$ \\
\hline \multirow[t]{5}{*}{ Edinburgh } & Kindia & $\begin{array}{l}\text { Stick dip }(6 ; \\
43 \%)\end{array}$ & $\begin{array}{l}\text { Leafy stick dip (6; } \\
43 \%)\end{array}$ & $\begin{array}{l}\text { Stick dip }(2 ; \\
50 \%)\end{array}$ & $\begin{array}{l}\text { Leafy stick dip }(2 ; \\
50 \%)\end{array}$ \\
\hline & Louis & $\begin{array}{l}\text { Stick dip }(6 ; \\
100 \%)\end{array}$ & & $\begin{array}{l}\text { Stick dip (13; } \\
87 \%)\end{array}$ & $\begin{array}{l}\text { *Leafy stick dip (2; } \\
13 \%)\end{array}$ \\
\hline & Lucy & $\begin{array}{l}\text { Stick dip (7; } \\
64 \%)\end{array}$ & Hand dip $(4 ; 36 \%)$ & $\begin{array}{l}\text { Stick dip }(2 ; \\
100 \%)\end{array}$ & \\
\hline & Kilimi & $\begin{array}{l}\text { Stick dip }(127 ; \\
73 \%)\end{array}$ & Hand dip $(33 ; 19 \%)$ & $\begin{array}{l}\text { Stick dip (49; } \\
48 \%)\end{array}$ & $\begin{array}{l}\text { Leafy stick dip (44; } \\
43 \%)\end{array}$ \\
\hline & Emma & $\begin{array}{l}\text { Stick dip }(19 ; \\
51 \%)\end{array}$ & Hand dip $(16 ; 43 \%)$ & $\begin{array}{l}\text { Stick dip }(38 ; \\
64 \%)\end{array}$ & $\begin{array}{l}\text { Hand dip }(8 ; 14 \%) / \\
* \text { Leafy stick dip }(8 ; \\
14 \%)\end{array}$ \\
\hline \multirow[t]{5}{*}{$\begin{array}{l}\text { Beekse } \\
\text { Bergen }\end{array}$} & Pearl & $\begin{array}{l}\text { Hand dip (165; } \\
53 \%)\end{array}$ & $\begin{array}{l}\text { Stick dip (134; } \\
43 \%)\end{array}$ & $\begin{array}{l}\text { Stick dip (119; } \\
56 \%)\end{array}$ & $\begin{array}{l}\text { Leafy stick dip (71; } \\
33 \%)\end{array}$ \\
\hline & Edith & $\begin{array}{l}\text { Stick dip }(320 ; \\
83 \%)\end{array}$ & Hand dip $(64 ; 17 \%)$ & $\begin{array}{l}\text { Stick dip }(364 ; \\
49 \%)\end{array}$ & $\begin{array}{l}* \text { Leafy stick dip } \\
(294 ; 40 \%)\end{array}$ \\
\hline & Eva & $\begin{array}{l}\text { Stick dip }(237 ; \\
76 \%)\end{array}$ & Hand dip $(61 ; 20 \%)$ & $\begin{array}{l}\text { Stick dip }(12 ; \\
57 \%)\end{array}$ & Hand dip $(5 ; 24 \%)$ \\
\hline & Frek & $\begin{array}{l}\text { Hand dip }(74 ; \\
59 \%)\end{array}$ & Stick dip $(27 ; 22 \%)$ & $\begin{array}{l}\text { Stick dip }(40 ; \\
50 \%)\end{array}$ & Stick drop $(23 ; 29 \%)$ \\
\hline & Rene & $\begin{array}{l}\text { Hand dip (141; } \\
93 \%)\end{array}$ & Stick dip $(7 ; 5 \%)$ & $\begin{array}{l}\text { Hand dip }(6 ; \\
55 \%)\end{array}$ & Stick dip $(3 ; 27 \%)$ \\
\hline
\end{tabular}

$6 \quad$ Note: Percentages are rounded. 


\section{Table 4(on next page)}

Results of full model GLMM on the effects of Phase, Subgroup and an interaction between the two upon 'Always effective' technique use. 
Table 4.

Results of full model GLMM on the effects of Phase, Subgroup and an interaction between the two, with random intercept and slope for Individual by Phase upon 'Always effective' technique use. Variance, standard deviation, and correlation for the random intercept and slope for Individual by Phase is provided.

\begin{tabular}{|c|c|c|c|c|c|c|}
\hline Fixed effects & Estimate & [Wald 95\% CI] & Std. Error & $z$ value & $p$ value & \\
\hline Intercept (including 'Wide & -4.13 & {$[-5.62,-2.64]$} & 0.76 & & & \\
\hline \multicolumn{7}{|l|}{ Tube' phase and Beekse } \\
\hline \multicolumn{7}{|l|}{ Bergen subgroup) } \\
\hline Phase ('Narrow Tube') & 2.93 & {$[1.15,4.70]$} & 0.91 & 3.23 & 0.0013 & $* *$ \\
\hline Subgroup (Edinburgh) & 1.57 & {$[-0.63,3.77]$} & 1.12 & 1.40 & 0.1631 & \\
\hline Phase*Subgroup & -1.57 & {$[-4.17,1.04]$} & 1.33 & -1.18 & 0.2387 & \\
\hline \multirow[t]{2}{*}{ Random effects } & Variance & & Std. & \multirow{2}{*}{\multicolumn{3}{|c|}{ Correlation }} \\
\hline & & & Deviation & & & \\
\hline Individual (Intercept) & 2.46 & & 1.57 & & & \\
\hline Slope by Phase & 3.31 & & 1.82 & & -0.87 & \\
\hline
\end{tabular}

$* *=p<0.01$ 